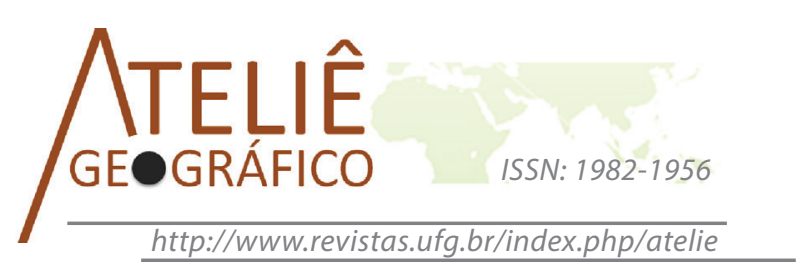

\title{
Técnicas de geoprocessamento aplicadas à análise de padrões pontuais como uma contribuição à cartografia de zonas sísmicas no estado de Goiás
}

\author{
Techniques GIS applied to the analysis of t points patterns \\ as a contribution to the cartography of seismic zones of the \\ state of Goiás
}

\section{Techniques de géotraitement appliquée à l'analyse des standards ponctuels comme une contribution à la cartographie des zones sismiques dans l'état de Goiás}

\author{
Alexandre Henrique Cardoso do Vale e Silva \\ Instituto Militar de Engenharia \\ alexandre.vale@ime.eb.br \\ Fabrizia Gioppo Nunes \\ Universidade Federal de Goiás \\ fabrizia.iesa.ufg@gmail.com
}

\begin{abstract}
Resumo
Eventos sísmicos estão em todo mundo, inclusive no Brasil. No Estado de Goiás, este fenômeno pode ser observado em determinadas regiões. O estudo sismológico é complexo, e se torna ainda mais, quando referente à tectônica intraplaca. $\mathrm{O}$ uso de técnicas de geoprocessamento, bem como da aplicação metodológica da análise espacial para o tratamento de registros sísmicos, podem contribuir para a interpretação destes eventos. Pretende-se aqui, entender os padrões pontuais na identificação de áreas de clusters de epicentros, representados em produtos cartográficos. A organização das informações de ocorrências sísmicas em ambientes de Sistema de Informação Geográfica colabora na análise dos fatos relacionados aos abalos tectônicos e de sua dinâmica, promovendo um diagnóstico para o zoneamento do potencial sismológico da região de estudo. Após análise dos mapas gerados em associação com a geologia estrutural e a geofísica, pode-se observar um aglomerado pertinente aos municípios das mesorregiões norte e noroeste do Estado.

Palavras-chave: Geotecnologia. Cartografia. Arranjo Espacial. Sismicidade Intraplaca.
\end{abstract}




\begin{abstract}
Seismic events are around the world, including Brazil. In the State of Goiás, this phenomenon can be observed in certain regions. The seismological study is complex, and it becomes even more so when referring to intraplate tectonics. The use of geoprocessing techniques, as well as the methodological application of spatial analysis for the treatment of seismic records, can contribute to the interpretation of these events. It is intended here, to understand the point patterns in identifying areas of clustering of epicenters, represented in cartographic products. The organization of seismic occurrence information on geographic information system environments collaborate on the analysis of facts related to tectonic quakes and its dynamics, promoting a diagnosis for the zoning of the seismological potential of the region studied. After analyzing the maps generated in association with structural geology and geophysics, one can observe a cluster relevant to the municipalities of the north and northwest mesoregions of the state.
\end{abstract}

Keywords: Geotechnology. Cartography. Spatial Arrangement. Intraplate Seismicity.

\begin{abstract}
Résumé
Événements sismiques sont partout dans le monde, y compris le Brésil. Dans l'État de Goiás, ce phénomène peut être observé dans certaines régions. L'étude sismologique est complexe, et devient encore plus lorsqu'on se réfère à la tectonique intraplaque. L'utilisation de techniques de géotraitement, ainsi que l'application méthodologique de l'analyse spatiale pour le traitement des enregistrements sismiques, peuvent contribuer à l'interprétation des événements. Il est prévu ici, comprendre les standards ponctuels dans l'identification des domaines des clusters d'épicentres, représentés dans les produits cartographiques. L'organisation des informations d'événements sismiques dans les environnements de système d'information géographique collabore sur l'analyse des faits liés aux tremblements de terre tectoniques et sa dynamique, en promouvant un diagnostic pour le zonage du potentiel sismique de la région d'étude. Après avoir analysé les cartes générées en association avec la géologie structurale et de la géophysique, on peut observer un cluster pertinente pour les municipalités des mésorégions du nord et du nord-ouest de l'État.
\end{abstract}

Mots-clés: Géotechnique. Cartographie. Arrangements Spatiaux. Sismicité Intraplaque.

\title{
Introdução
}

De acordo com o contexto estrutural geológico brasileiro a maioria dos abalos sísmicos, são raros de serem sentidos pela população, porém casos de magnitude $>6,0$ graus na escala Richter já ocorreram no Brasil.

O estudo sismológico se faz necessário para o entendimento maior das camadas do interior da Terra, compreendendo os impactos que as ondas de choque provocam na superfície. Não devemos esquecer que as estruturas que suportam a superfície terrestre, promovem uma variedade de feições geoestruturais, onde suas propriedades merecem uma atenção, a priori, de qualquer ação de grade porte sobre o relevo. 
Ao observamos a dinâmica sísmica de Goiás, estimula-se uma reflexão a respeito das condições geofísicas locais. Os registros sismológicos coletados no Observatório Sismológico da Universidade de Brasília (UnB), no período de 1973 à 2013, tem demostrado um número considerável de abalos sísmicos nas mesorregiões do norte e noroeste goiano, em relação as outras mesorregiões do estado, porém de magnitude moderada, $<5,2$ graus na escala Richter.

É relevante analisarmos as regiões em destaque, interpretando os dados geotectônicos e geofísicos, na visão da distribuição de padrões espaciais, da ocorrência deste fenômeno, como uma corroboração ao desenvolvimento de modelos que buscam explicações para ocorrência de terremotos no Brasil.

A partir do momento que há um acréscimo populacional, somam-se também os problemas observados pelo homem, logo, não é diferente se tratando dos terremotos que ocorrem em regiões de tectônica relativamente estável.

Em Goiás os maiores tremores registrados foram os que ocorreram em 2010 no município de Estrela do Norte e em 2012 no município de Mara Rosa ( $5^{\circ}$ na escala Richter), localizado na microrregião de Porangatu no norte goiano. Em noticiários locais e até mesmo em relevância nacional, o testemunho dos habitantes mara-rosenses e de municípios vizinhos, destaca a preocupação do tremor diante de um fenômeno raro (PESQUISA-FAPESP, 2013).

Além do pânico em alguns municípios do norte-noroeste goiano, as ondas se espalharam em todo o globo, sendo registradas em vários sismógrafos do mundo e até mesmo percebidas, pelos habitantes da capital do país, com a evacuação de alguns prédios públicos, documentada pela imprensa na ocasião.

Um estudo sismológico periódico faz com que se interpretem as condições geofísicas da região em destaque. A análise das disposições locacionais de ocorrência dos epicentros sísmicos, permite compreender melhor o arrojo espacial destes abalos, instigando um estudo mais detalhado dos aspectos geoestruturais no âmbito deste recorte espacial.

Enfoca-se, que o posicionamento dos fenômenos terrestres é de fundamental importância para relacionarmos os acontecimentos ao um local. Na visão de Longley et al. (2013), problemas que envolvem um aspecto de localização, são denominados problemas geográficos. Segundo os autores, com o surgimento das técnicas de geoprocessamento e suas ferramentas computacionais a tarefa de organizar os dados, assim como, reconhecer os problemas geográficos, fica mais fácil.

Nesse termo, os SIGs, consisti um exemplo dessas técnicas que tem apresentado um amplo auxílio para o tratamento de informações espaciais, fazendo parte do universo geotecnológico, que caracteriza a organização das informações geográfica. Com isso a relação da paisagem real é transformada em paisagem digital (LONGLEY et al., 2013).

Para uma análise espacial de dados geográficos é necessário um fenômeno, onde este tenha uma localização no espaço. Tais elementos podem ser examinados de acordo 
com métodos de análises espaciais que permitem a compreensão mais concreta dos fatos determinados.

Segundo Câmara et al. (2001), existem técnicas computacionais que analisam tais fenômenos em relação aos dados espaciais, materializando as informações em produtos cartográficos. Essas produções cartográficas irão representar de maneira sistemática a organização dos dados em seu nível de observação, no que diz respeito às informações obtidas, logo, caracteriza a realidade para um exame detalhado objetivando o planejamento em várias áreas de conhecimento.

São considerados três tipos de dados relacionados a problemas de analise espacial: (a) Áreas com Contagens e Taxas Agregadas; (b) Superfícies Contínuas e; (c) Eventos ou Padrões Pontuais, sendo este último à localização espacial do fenômeno, como o fator principal de análise associados a padrões aleatórios ou aglomerados das disposições pontuais (CÂMARA et al., 2001).

Nesta perspectiva, o estudo ora proposto, da distribuição espacial em plataformas SIG, permite a interpretação mais coerente do fenômeno analisado, comportando um nível de observação onde às variáveis presentes são organizadas de acordo com a proposta da pesquisa, que propende à compreensão das aglomerações das ocorrências sísmicas no estado de Goiás.

No intuito de atender tal proposta, o presente trabalho, objetiva a análise da distribuição de arranjos espaciais da ocorrência de eventos sísmicos, reconhecendo áreas de cluster a partir do emprego da técnica de Kernel, na identificação de padrões pontuais, bem como a correlação destes resultados com informações geológicas e geofísicas existentes.

\section{Contexto geoestrutural}

Para entender a sismicidade brasileira, é importante compreender o contexto estrutural geológico do país. O Brasil está inteiramente localizado na plataforma SulAmericana de idade pré-cambriana. Uma porção relativamente estável dividida em escudos pré-cambrianos e coberturas fanerozóicas, localizada a leste da Cadeia Andina, que corresponde à área mais instável do continente.

Os embasamentos pré-cambrianos são escudos cristalinos caracterizados por rochas ígneas e metamórficas e são relativamente estáveis se tratando da tectônica. Esses embasamentos são atribuídos no conceito geológico, como porções da litosfera constituídas por substratos de origem arqueano e proterozóico. Já as coberturas fanerozóicas são estruturas sedimentares intracratônicas, herdadas do ciclo BrasilianoPan-Africano (MILANI \& THOMAZ FILHO, 2000).

A dinâmica geológica estrutural do Brasil faz com que a atividade sísmica presente no país, seja muito inferior aos abalos ocorridos nas bordas das placas, por exemplo, no contato entre as placas de Nazca e a placa Sul-Americana, porém, isso não quer dizer que a região seja assísmica. Devido a sua localização na porção central 
da placa da América do Sul, a sismicidade presente no território brasileiro é de origem intraplacas.

Explicar as origens de sismos intraplacas não é uma tarefa fácil. Zonas de fraqueza e concentração de tensões são alguns modelos propostos na tentativa de uma compreensão dessa atividade. É sabido que os abalos ocorridos nesses locais, em sua maioria, têm baixa e moderada intensidade, são decorrentes das tensões geradas por parte dos sismos tectônicos originados entre as fronteiras das referidas placas (JOHNSTON \& KANTER, 1989).

Todavia, alguns sismos podem ter grande magnitude como no caso de Porto dos Gaúchos no Mato Grosso com magnitude de 6,2 graus e em Vitória no Espírito Santo com magnitude de 6,1 graus, ambos no ano de 1955 registrados por pesquisadores do exterior, pois, naquela época praticamente não existiam registros sismológicos no país.

Em estudos relacionados à sismicidade intraplacas na região do Brasil Central, Assumpção e Sacek (2013) verificaram que onde a crosta terrestre é mais fina, os abalos sísmicos se concentram. Em suas pesquisas, os autores destacam que por intermédio de observações de modelagens das anomalias gravimétricas no Brasil Central, supõem-se que, o excesso de massa, provocadores das anomalias isostáticas é devido a variações de espessura crustal.

Segundo os autores a crosta estreita ocorre ao longo da zona do arco magmático do Maciço de Goiás, fazendo um paralelo com a zona de alta intensidade do campo gravitacional, indicando que ali, há o espessamento do manto superior, promovedor de uma zona sísmica tensionada (ASSUMPÇÃO \& SACEK, 2013).

Em referência ainda ao Brasil Central Lopes (2003), apresenta um interessante estudo da estrutura crustal média da região de Goiás, com a minimização dos resíduos de determinações hipocentrais. Como resultado o autor obteve uma crosta com espessura média de 38,0 km. Porem estudos gravimétricos mostra que em algumas regiões, a crosta, pode chega a ser mais fina do que $35 \mathrm{~km}$, no sudeste do Mato Grosso e noroeste goiano.

Berrocal et al. 2004, pesquisando dados de refração sísmica por modelagem gravimétrica na região Central da Província de Tocantins, já haviam delineado algumas altas e baixas anomalias crustais, separadas por um gradiente íngreme com direção SWNE. Segundo os autores, a faixa sísmica Goiás-Tocantins, parece estar espacialmente associada a uma forte anomalia gravimétrica, interpretada como uma sutura com espessamento crustal na parte leste. Nesta parte do globo é verificada que, a profundidades para Moho variam de 32 a 43 km, sob o lado ocidental do Arco Magmático de Goiás.

Nas literaturas geológicas, referentes a sismos no Brasil, já é consenso que a Faixa Sísmica Goiás-Tocantins é uma notável feição sismotectônica dentro da Província Tocantins. Segundo Lopes (2008) a faixa sísmica atravessa parte do Maciço de Goiás e parte dos dobramentos Brasilianos.

Segundo Ventura et al. (2011) a porção da Zona Sismológica de Porangatu, região dos terrenos do Arco Magmático de Goiás e do Maciço de Goiás, também 
marcada pelo alto gravimétrico Bouguer do Brasil central é uma anomalia regional, com aproximadamente $200 \mathrm{~km}$ de largura e $700 \mathrm{~km}$ de extensão na direção NE, caracterizada por valores de anomalia Bouguer entre $+10 \mathrm{mGal}$ e $-10 \mathrm{mGal}$, que contrastam com valores entre $-60 \mathrm{mGal}$ e $-70 \mathrm{mGal}$ a $\mathrm{NW}$ e entre $-100 \mathrm{mGal}$ e $-140 \mathrm{mGal}$ a SE.

\section{Materiais e método}

\section{Estimador de Kernel}

$\mathrm{Na}$ análise espacial das ocorrências sísmicas foi utilizado o estimador de intensidade de Kernel, que possibilita a estimação da densidade do evento em toda a área de interesse, mesmo em regiões onde o processo não tenha gerado nenhuma ocorrência real. Procura estimar a partir da ocorrência de eventos em áreas vizinhas a probabilidade, ou seja, o grau de risco de ocorrência do evento em cada unidade de área da dimensão espacial analisada.

É considerado, por tanto, como um estimador estatístico probabilístico e não paramétrico, ou seja, consiste em estimar o número esperado de eventos por unidade de área. A Figura 1 representa o princípio esquemático do estimador de intensidade de Kernel.

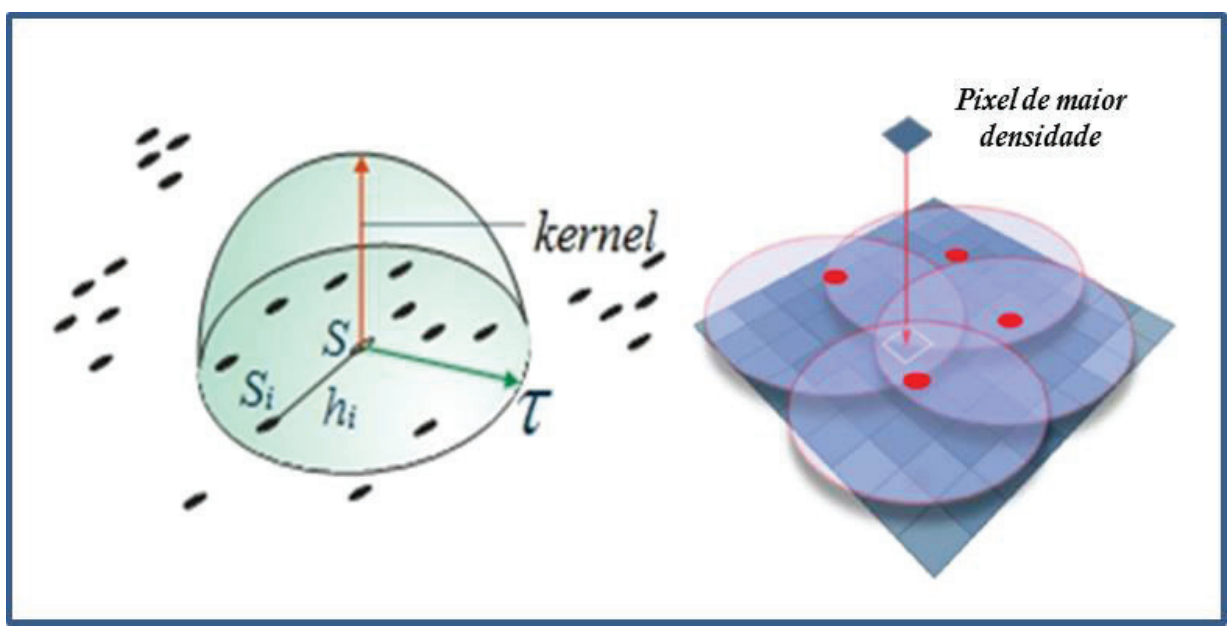

Figura 1: Estimador de Densidade de Kernel.

De acordo com Matsumoto e Flores $(2012,96)$ o estimador de Kernel é uma técnica de estatística espacial que demonstra onde estão alocadas as concentrações de eventos pontuais em um plano. Quando este estimador é utilizado em um SIG, gera uma interpolação que se insere sobre as bases cartográficas, identificando o fenômeno georreferenciado no espaço geográfico e, destacando, a localização em que ocorre a maior ou menor frequência das concentrações do fenômeno analisado. 
Em suma, o método de Kernel consiste em uma função bidimensional, a qual se define uma largura de banda, fazendo-se a contagem de pontos dentro da região de influência, ponderados pela distância de cada um à localização de interesse. Como produto final, os resultados estarão arranjados em um plano cujo valor será proporcional à frequência de amostras por unidade de área, obtendo os cálculos quanto ao seu valor de densidade (BAILEY \& GATRELL, 1995).

\section{Procedimentos técnicos}

No trabalho foram utilizados os Sistemas de Informação Geográfica (SIGs), na plataforma $A R C G I S^{R}$ e TerraView, além das bases cartográficas vetoriais, coletadas do banco de dados do Instituto Brasileiro de Geografia e Estatística (IBGE), sendo essas bases, referentes ao limite estadual e dos munícipios de Goiás. Para as estruturas geológicas foram usados dados do Serviço Geológico do Brasil - Companhia de Pesquisas em Recursos Minerais e UnB (CPRM/METAGO/UnB, 2000). Essas informações permitiram a localização geotectônica da área de estudo.

Para a verificação dos eventos sismológicos foram utilizados dados sísmicos, em arquivos digitais no formato shapefile, cedidos pelo Observatório Sismológico da UnB. Os dados do observatório descrevem as informações dos abalos sísmicos no estado de Goiás, entre o período de 1973 até 2008. Foi utilizada também a compilação de ocorrências sísmicas do Websisbra, um Sistema de Informação Geográfica Web desenvolvido pela UnB, para a Análise de Fenômenos Sismológicos além de dados contidos no Boletim Sismológico do Instituto de Astronomia, Geofísica e Ciências Atmosféricas (IAG/USP), objetivando, completar os registros até o ano 2013. O levantamento foi contemplado por dados disponíveis em literaturas sobre o estudo de métodos tomográficos do IAG/USP, para obter informações das propriedades físicas da região do Brasil Central.

No decorrer das análises, para aplicação mais detalhada do estimador de Kernel, foi utilizada a ferramenta de análise espacial, Mapa de Kernel, do aplicativo TerraView que permite o cálculo da função Quártica para a análise da: (a) densidade, (b) média de movimento espacial e (c) probabilidade dos eventos pontuais com o atributo magnitude ativo.

A função Quártica dá mais peso aos pontos próximos do que aos distantes, sendo o decréscimo gradual. O aplicativo TerraView, possibilita a opção de adaptar o raio do círculo de vizinhança, ou seja, durante o processamento foi utilizada a opção Kernel Adaptativo. Essa alternativa calcula automaticamente o raio, considerando o número de eventos e a dimensão da unidade de área avaliada.

A ferramenta computacional não considera apenas a estimativa da distribuição da densidade espacial, mais possibilita também, o cálculo do peso da magnitude dos eventos, ou seja, o cálculo do peso da desigualdade de importância dos sismos na escala Richter.

Para efeito de zoneamento de risco, foi calculada a intensidade do evento por unidade de área, sendo as delimitações municipais, a unidade de área territorial, 
selecionada neste estudo para a captura e representação dos dados quantitativos. A Figura 2 ilustra o esquema de aplicação da ferramenta Mapa de Kernel, do software TerraView, utilizada na elaboração dos mapas de riscos de sismo por municípios.

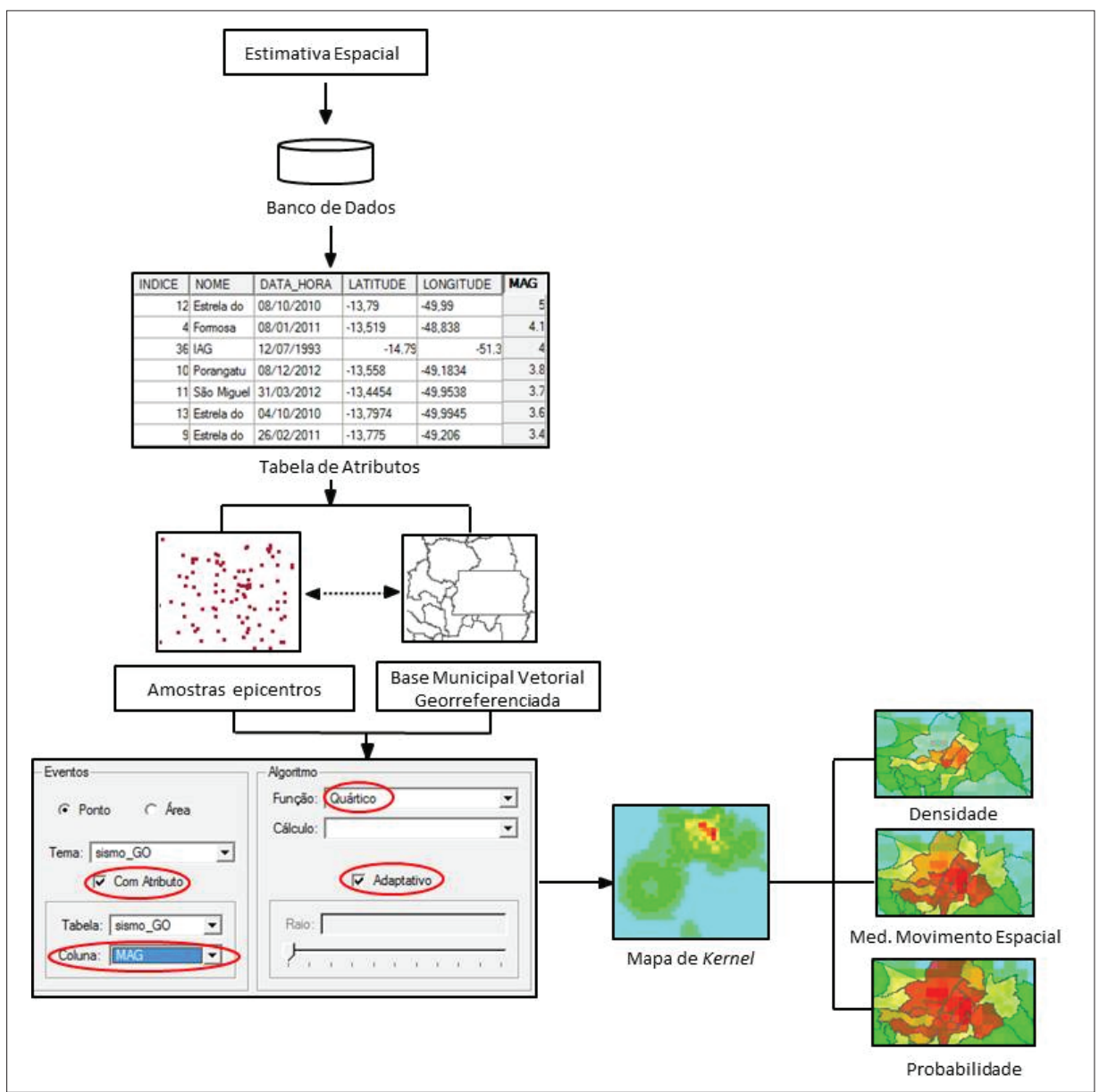

Figura 2: Esquema utilizado pelo estimador de Kernel, no processamento de análise espacial dos pontos de epicentros registrados na Estação Sismológica da UnB, entre o período de 1973 a 2013.

\section{Resultados e discussões}

O tratamento das informações sismológicas do estado de Goiás foi realizado visando à caracterização espaço-temporal da sismicidade e sua correlação com as estruturas geológicas e geofísicas locais, de forma a obter a configuração do potencial sísmico da região, nos últimos 40 anos. Ao compilar os dados do observatório sismológico 
foi gerado um produto cartográfico, representado pela Figura 3, que possibilita visualizar a distribuição e o grau de magnitude na escala Richter, das ocorrências dos eventos sísmicos, por mesorregiões do estado de Goiás.

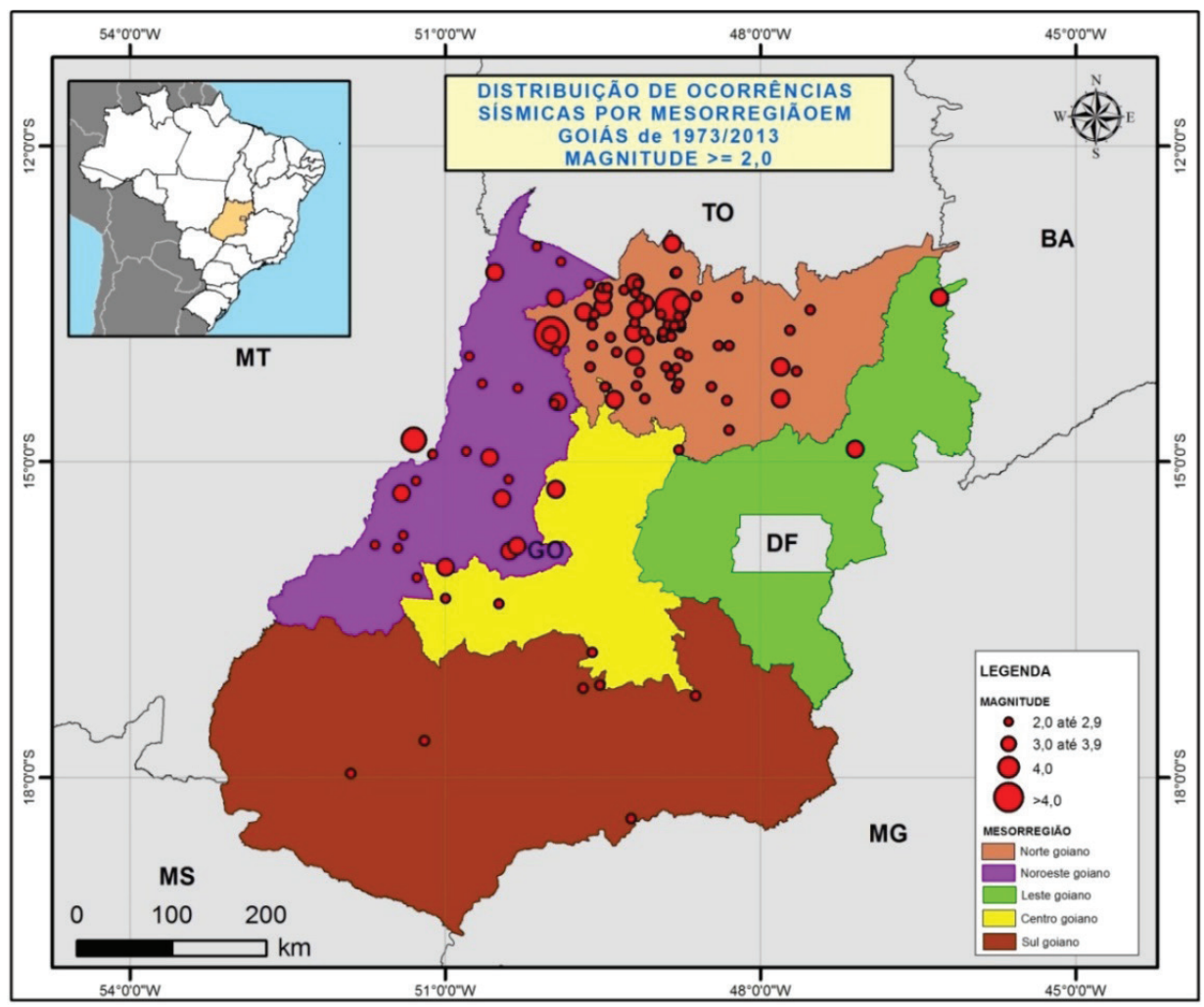

Figura 3: Distribuição espacial da magnitude e das ocorrências sísmicas nas mesorregiões do estado de Goiás, entre 1973 a 2013. Os círculos em vermelho representam os sismos com magnitude maior que 2,0 na escala $\mathrm{mR}$.

Da derivação das informações contidas na Figura 3 interpreta-se que as regiões leste, central e sul do estado de Goiás não concentram muitos registros de abalos geotectônicos intraplacas, concentrando a densidade de registros e os maiores peso da magnitude sísmica, nas mesorregiões noroeste e norte, em especial, nesta última.

É importante ressaltar os sismos de $5^{\circ}$ de magnitude na escala Richter, ocorridos em 2010 e 2012 na região de Porangatu, norte goiano. Para a questão sismológica local, e brasileira, estes eventos tornam-se importantes porque despertaram o interesse da comunidade científica, para estudos mais detalhados da Zona Sísmica Goiás-Tocantins.

Ao processar os dados em ambiente SIG utilizando técnicas de geoprocessamento, para a análise espacial da densidade de Kernel, foi gerado um produto cartográfico, enfocando, as principais regiões de intensidade sísmica (Figura 4). Como resultado deste 
produto, obteve-se o delineamento de uma área de cluster, no âmbito do território de Goiás e, no que se refere às estruturas geológicas da região.

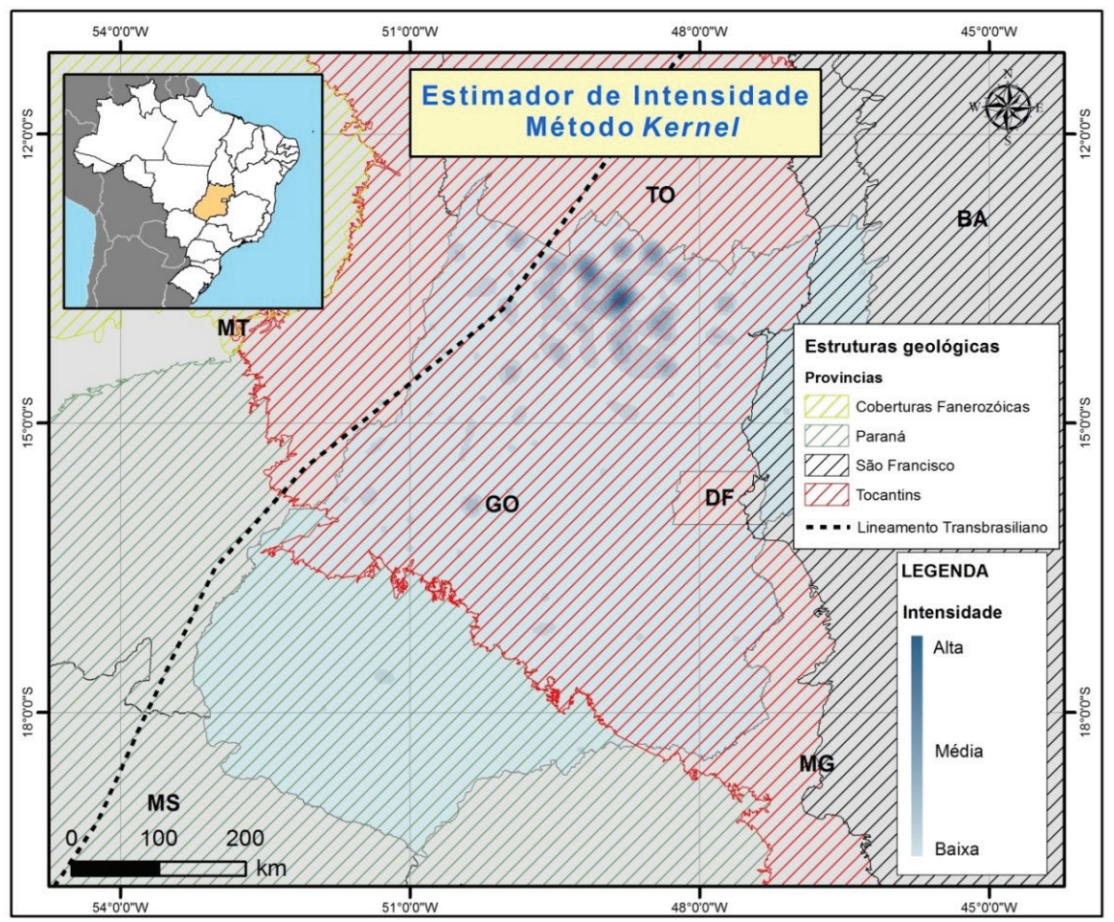

Figura 4: Mapa de densidade sísmica determinada pelo estimador de Kernel e estruturas geológicas do estado de Goiás.

Da organização destes dados, observa-se que a disposição das ocorrências sísmicas no estado, apresenta-se com grau de intensidade mais alta na porção norte, sobre a estrutura geológica da Província Tocantins, ou seja, na Zona Sísmica de Porangatu.

Dentro da caracterização do contexto geológico presente, as ocorrências destes sismos estão representadas em toda a província Tocantins, mais precisamente nas bordas do lineamento transbrasiliano, que corresponde a uma zona de fraqueza litosférica, com cerca de $2.700 \mathrm{~km}$ de extensão no território brasileiro. Nesta zona de fraqueza, concentram-se os esforços gerados nos limites das grandes placas tectônicas, onde em tempo futuro são liberadas as tensões, sendo um potencial para sismicidade intraplacas.

Nota-se também a proximidade da zona sísmica Goiás-Tocantins com a intensidade das ocorrências na região. Um paralelismo com o lineamento transbrasiliano com a zona citada. A localização da mesma pode ser observada na Figura 4.

O resultados da aplicação do processo estocástico de interpolação dos pontos de epicentros, pelo estimador de Kernel, utilizando as ferramentas computacionais do 
aplicativo TerraView, podem ser visualizados nos mapas da Figura 5, que representam o diagnóstico e prognóstico da intensidade de zonas de rico, em âmbito municipal.

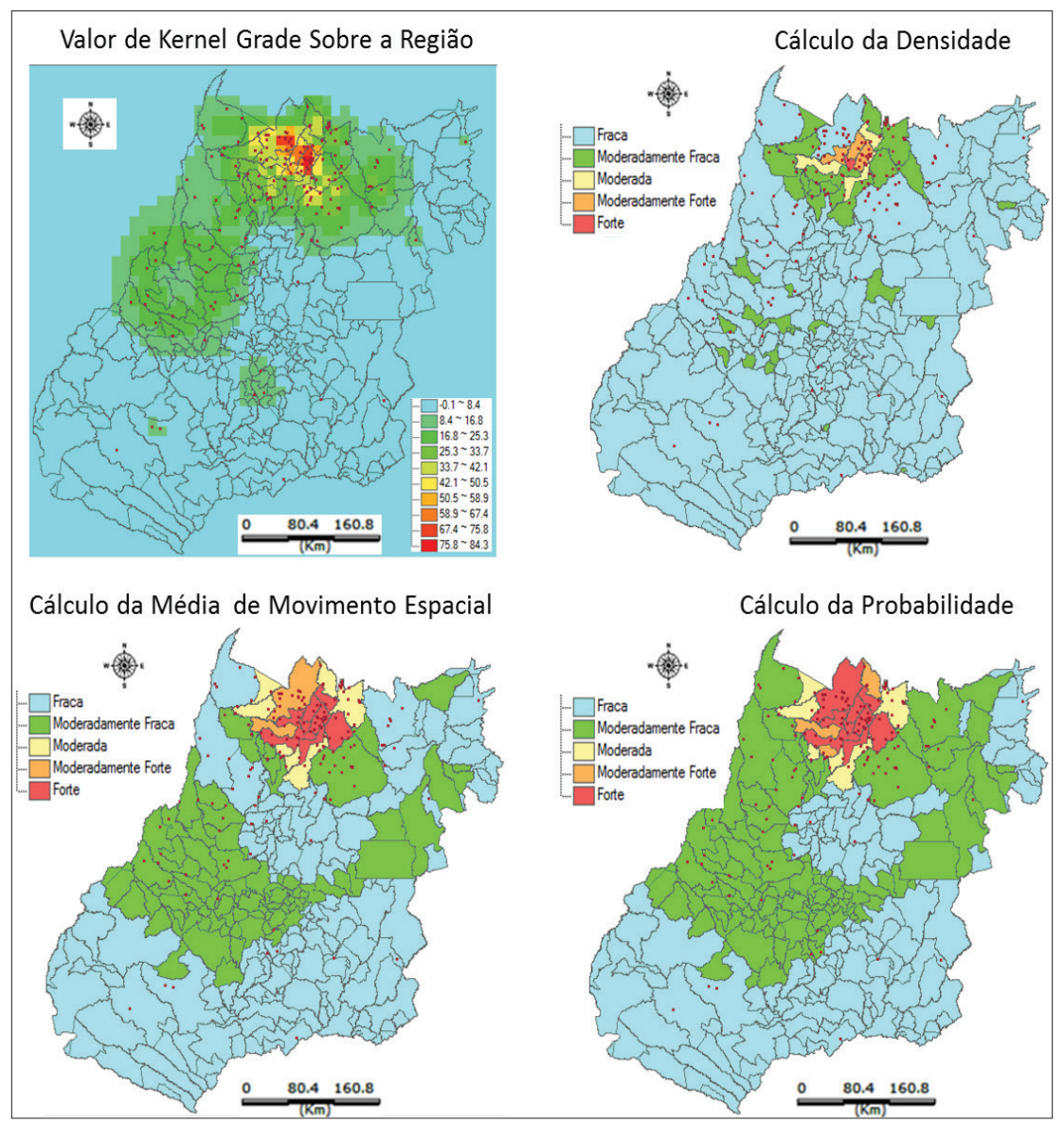

Figura 5: Mapas derivados da aplicação do estimador de Kernel (função Quártico), dos pontos de epicentros registrados na estação sismológica da UnB, entre o período de 1973 a 2013.

No caso referente à concentração dos eventos sísmicos, nos municípios goianos verifica-se que tanto para os cálculos de densidade, média de movimento espacial e de probabilidade, há a formação de cluster de forte intensidade sobre os municípios pertencentes à porção norte do estado.

Em ambos os mapas o comportamento dos padrões pontuais representa um agrupamento ou interação espacial, das classes de valores da densidade de Kernel. Agrupamento este visualizado no mapa de Média de Movimento Espacial, representado pelos municípios de Estrela do Norte, Formoso, Santa Tereza de Goiás, Mutunópolis, Trombas, Campinaçu, Campinorte e Mara Rosa. Na representação da média de 
movimento espacial, a classe de intensidade forte, representada pelos municípios citados, indica um alto número esperado de sismos nestas unidades de área, em comparação aos demais municípios do estado.

Já o mapa da Probabilidade de Kernel, indica a probabilidade de um sismo que já ocorreu, estar localizado em determinada área. Neste mapa verificamos esta alta probabilidade, indicada no cluster da classe de intensidade forte, formada pelos mesmos municípios apontados no mapa de Média de Movimento Espacial, acrescido pelo município de Porangatu.

Por fim, o mapa de Densidade de Kernel, indica a densidade de sismo por unidade de área, indicando um ponto focal da classe de intensidade forte, representada sobre o município de Estrela do Norte, procedida pela classe moderadamente forte constituída pelos municípios de Santa Tereza de Goiás, Mutunópolis e Formoso.

Quanto aos dados tomográficos associados à crosta fina, onde é previsto um potencial para a concentração de tremores, estes podem ser observados na interpretação cartográfica da Figura 6. Esses dados juntamente com os registros sísmicos plotados, evidenciam uma densidade maior de eventos sísmicos na região norte-noroeste de Goiás, onde a crosta é menos densa, ou seja, na Zona Sísmica de Porangatu.

A ilustração vêm a corroborar com as análises das anomalias gravimétricas realizadas por Assumpção \& Sacek (2013), delineadas pela zona sísmica de alta intensidade do campo gravitacional, concomitantes ao estreitamento crustal e aumento de densidade da crosta inferior, mantendo assim, o princípio da isostasia.

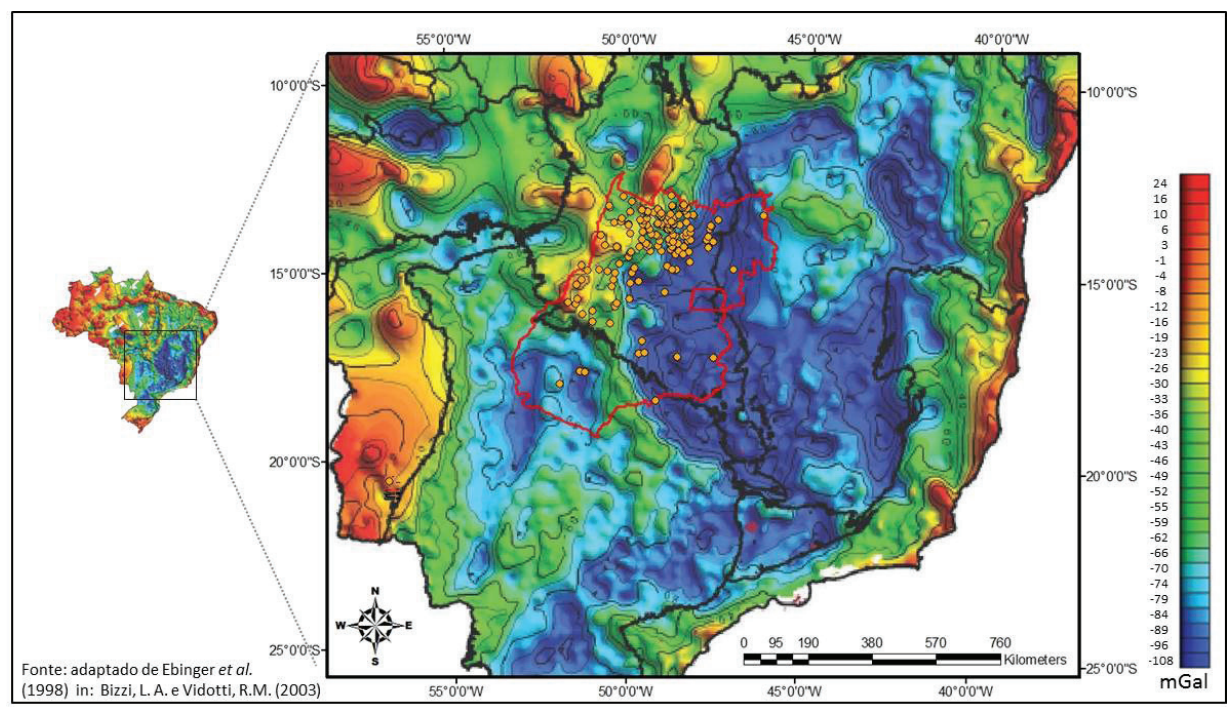

Figura 6: Mapa de anomalias gravimétricas de Bouguer com base em um grid de ca. $5 \mathrm{~km}$ e, integrado com a delimitação territorial do estado de GO (em vermelho) e os pontos de eventos sísmicos (em laranja), registrados pela estação sismológica da UnB (1973 a 2013). 


\section{Considerações finais}

A partir das técnicas de geoprocessamento associada a análise espacial, pôde-se obter uma melhor compreensão da dinâmica dos padrões focais sísmicos relacionados a uma zona, com densidade maior do que, em outras regiões goianas, onde este evento não se faz tão presente. Estas técnicas permitiram averiguar a probabilidade de ocorrência e magnitude deste fenômeno, em um determinado local, e sua distribuição no espaço, em um período de 40 anos.

As técnicas de geoprocessamento associada à análise espacial possibilitaram também, a ordenação quantitativa dos dados espaciais, promovendo o potencial analítico dos fenômenos sísmicos, registrados na área de estudo em questão.

Os dados coletados e processados mostraram-se bastante relevantes, para um melhor entendimento da dinâmica e do zoneamento do potencial sismológico do estado de Goiás, evidenciando como os abalos sísmicos se distribuem em todo o estado, em termos de densidade e em peso de magnitude.

As análises dos produtos cartográficos, mostraram que há uma disposição sismológica maior para porção setentrional goiana, apresentando uma zona sísmica de eventos pontuais nesta região, coincidente com a já reconhecida Zona Sísmica de Porangatu, formada pelos municípios pertencentes à microrregião de Porangatu.

Essas informações nos remetem à conclusão que, o método de análise espacial aplicado, está diretamente subordinado a consistência e precisão dos dados analisados e mapeados. Desta forma pode-se jugar, que de posse de dados confiáveis, processamento adequado e interpretação coerente, os problemas geográficos podem sim serem analisados para um entendimento mais detalhado, no que diz respeito às condições geoestruturais.

Não podemos esquecer que os produtos cartográficos gerados, são capazes apenas de determinar a probabilidade de ocorrência de um terremoto em uma área particular, com base somente no quantitativo de eventos que ocorreram no passado.

Não menos relevante, as ferramentas de análise espacial do aplicativo TerraView, possibilitaram a determinação de distintas zonas, delineadas por um recorte territorial municipal, apontando aglomerados de municípios com zonas de intensidade forte, à aglomerados de municípios com zona de fraca intensidade.

Logo, o estudo sísmico é importante para evitar futuros desastres, onde a catástrofe é caracterizada pelo dano sofrido no meio social. Identificar zonas, onde os tremores são comuns, é uma responsabilidade científica por uma atenção maior, e assim, evitando calamidades que põe em risco a vida de civis.

\section{Agradecimentos}

Ao Observatório Sismológico da Universidade de Brasília (UnB), pelo fornecimento dos dados sismológicos usados na pesquisa, bem como o centro sismológico da Universidade de São Paulo (USP). 


\section{Referências}

ASSUMPÇÃO, M.; SACEK, V. (2013). Intra-plate Seismicity and Flexural Stresses in Central Brazil. Geophysical Research Letters. American Geophysical Union, v. 40, n. 3, p. 487-491.

BAILEY, T. C.; GATRELL, A. C. (1995). Interactive Spatial data Analysis. Harlow Essex, England: Longman Scientific and Technical, 413p.

BERROCAL, J; MARANGONI, Y.; FUCK, R.; SOARES, J. E. P.; DANTAS, E.; PEROSI, F.; FERNANDES, C. (2004) Deep seismic refraction and gravity crustal model and tectonic deformation in Tocantins Province, Central Brazil. Tectonophysics. International Journal of Geotectonics and the Geology and Physics of the Interior of the Earth, v. 388, n. 1, p. 187-199.

BIZZI, L. A.; VIDOTTI, J. H. (2003). Condicionamento do magmatismo pós-Gondwana. In. Bizzi, L. A.; Schobbenhaus, C.; Vidotti, R. M.; Gonçalves, J. H. (ed.). Geologia, tectônica e recursos minerais do Brasil: texto, mapas \& SIG. Brasília: CPRM Serviço Geológico do Brasil, cap. VI, p.335-362.

CÂMARA, G.; MONTEIRO, A. M; DAVIS, C. (2001). Introdução à Ciência da Geoinformação. São José dos Campos. Disponível em:http://www.dpi.inpe.br/gilberto/ livro. Acesso em: 14 de set. de 2013.

IAG (2001-2013). Instituto de Astronomia, Geofísica e Ciências Atmosféricas. Boletim Sísmico Brasileiro. Catálogo do IAG/USP. Disponível em: http://moho.iag.usp.br/ sismologia/boletim/boletim2001p.txt. Acesso em jul. 2013.

JOHNSTON, A. C; KANTER. L. R. (1989). The seismicity of stable continental interiors. In: Gregersen, S. \& Basham, P.W. Earthquakes at North-Atlantic Passive Margins: Neotectonics and Post-glacial Rebound. Boston, EUA: Kluwer Academic, p.299-327.

LONGLEY, P. A.; GOODCHILD, M. F.; MAGUIRE, D. J.; RHIND, D. W. (2013). Sistemas e Ciência da Informação Geográfica. Tradução: Schneider, A. et al. 3a . ed. Porto Alegre: Bookmen, 540p.

LOPES, A. E. de V. Sismicidade e Estrutura Curstal em Goiás. (2003). Monografia (Trabalho de graduação). Instituto de Astronomia, Geofísica e Ciências Atmosféricas, Departamento de Geofísica - USP, São Paulo, 2003.

LOPES, A. E. DE V. (2008). Mecanismos focais e esforços litosféricos no Brasil. Tese (Doutorado). Instituto de Astronomia, Geofísica e Ciências Atmosféricas, Departamento de Geofísica - USP, São Paulo, 2008.

MATSUMOTO, P. S. S.; FLORES, E. F. (2012) - "Estatística espacial na geografia: um estudo dos acidentes de trânsito em Presidente Prudente - SP". GeoAtos - Revista geografia em Atos, Vol. 1, $\mathrm{N}^{\mathrm{o}}$ 12, 95-113. Disponível online no endereço url:http:// revista.fctunesp.br/index.php/geografiaematos/article/viewFile/1755/matsumoto (acedido em 10 de Maio, 2014). 
MILANI, E. J.; THOMAZ FILHO, A. (2000) Sedimentary Basins of South America. In: Cordani, U.G.; Milani, E. J.; Thomaz Filho, A; Campos, D.A. (org.). Tectonic Evolution of South America. Rio de Janeiro, 31st. IGC, p. 389-449.

PESQUISA FAPESP. (2013). Pesquisadores buscam as razões para a terra tremer no Brasil. Revista de Pesquisa da FAPESP. Disponível em:http://revistapesquisa.fapesp. br/2013/07/19/pesquisadores-buscam-as-razoes-para-a-terra-tremerno-brasil/. Acesso em: out. 2013.

CPRM/METAGO- Programa Levantamentos Geológicos Básicos do Brasil. Geologia e Recursos Minerais do Estado de Goiás e do Distrito Federal. (2000). Escala 1:500.000. Filho, J. V. L. de; Rezende, A. Silva, A. da. $2^{\text {a }}$ ed. Goiânia: CPRM/ METAGO/UnB, 2000.

SAATKAMP, H. D. (2013) Websisbra - Sistema Nacional de Registros Sísmicos. Monografia (Trabalho de graduação), Departamento de Ciências da Computação, UnB, Brasília, 2013.

VENTURA, D. B. R.; SOARES, J. E. P.; FUCK, R. A.; CARIDADE, L. C. C DA. (2011). Caracterização sísmica e gravimétrica da litosfera sob a linha de refração sísmica profunda de Porangatu, Província Tocantins, Brasil Central. Revista Brasileira de Geociências. v. 41, n. 1, p. 130-140.

Alexandre Henrique Cardoso do Vale e Silva

Graduado em Geografia pelo Instituto de Estudos Sócio-Ambientais da Universidade Federal de Goiás e mestrando no Instituto Militar de Engenharia PG SE/6 Engenharia Cartográfica.

Praça General Tibúrcio, 80 - Praia Vermelha - Urca - Rio de Janeiro - RJ - 22290270

alexandre.vale@ime.eb.br

Fabrizia Giopro Nunes

Doutora em Geologia Ambiental pela Universidade Federal do Paraná e professora efetiva do Instituto de Estudos Socioambientais da Universidade Federal de Goiás.

Campus Samambaia Caixa Postal 131. Goiânia - GO

CAMPUS II Samambaia - Cx. POSTAL 131 - Goiânia - GO / CEP: 74001-970

fabrizia.iesa.ufg@gmail.com

Recebido para publicação em junho de 2015 Aprovado para publicação em setembro de 2015 